\title{
The Influence of Chinese Social Behavior Orientation on Chinese-English Translation of Trademarks
}

\author{
Yonghong Wu \\ Xi'an University, Shaanxi, China, 710065
}

Keywords: Chinese social behavior orientation; Vision fusion; trademark translation

Abstract: With the globalization of economy and the deepening of China's reform and opening-up to the outside world, more and more Chinese products are being sold around the world, and trademark translation has become an important part of translation activities. The Chinese social behavior orientation has the distinctive characteristics of others' orientation, which inevitably affects the translator's translation activities and guides the translator's translation thoughts and actions to a certain extent. The realization of "vision fusion" is the reflection of others' guiding role in the translation of Chinese and English trademarks. This paper briefly introduces the orientation of others in the social behavior orientation of Chinese and the "scope fusion" under the guidance of others. It mainly analyzes the role of "view fusion" under the orientation of others in trademark translation, in order to continuously improve the quality of Chinese-English translation of trademarks.

\section{Introduction}

The famous scholar Lisman once divided the national character into three types: intrinsic orientation, others-oriented and traditional orientation. Among them, the orientation of others refers to "the goal pursued changes with the orientation, and only the process of pursuing the process itself and paying close attention to the behavior of others will remain unchanged for the rest of their lives". Trademark translation is a special form of translation. The original trademark is only one of the aspects of translation considerations and does not necessarily correspond to the content. The pursuit of trademark translation is that the translation achieves the same effect as the original text. "Horizontal Fusion" regulates the whole process of trademark translation ${ }^{[1]}$.

\section{The orientation of others in Chinese social behavior orientation}

Some scholars have studied the Chinese people's social behavior orientation and found that "Chinese people have obvious guiding qualities in the choice of social behavior". This kind of other people's orientation may be active or may be forced to make choices. The orientation of others emphasizes the thoughts, speeches, and behaviors of others, and accordingly, when it comes to the behavior of others, it is also susceptible to others, and this concern for others is continuous. American scholar Lisman pointed out that the orientation of others is easy to form a succession, which is "to maintain a certain sensitivity to the actions and wishes of others". The formation of this kind of personality orientation in China is closely related to China's long-standing social culture, 
values, religious beliefs and other factors. Figure 1 is the main factor affecting the orientation of Chinese people to form a social orientation of others ${ }^{[2]}$.

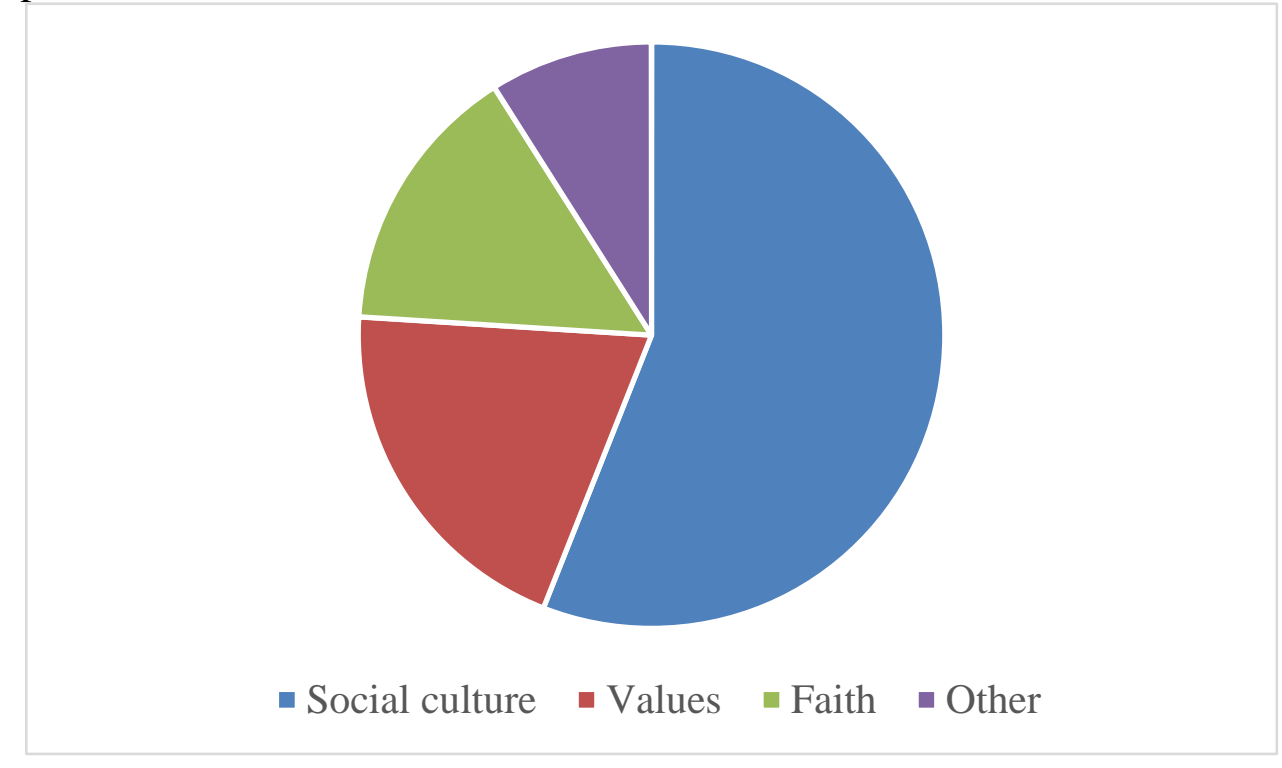

Figure 1 Reasons for the formation of Chinese social behavior orientation

\section{3. "View fusion" under the guidance of others}

The Chinese people pay attention to the obvious behavioral characteristics of others, so that when the translator is translating, he or she consciously or unconsciously pays more attention to the aesthetic psychology and values of the target readers, and the desire to make their translations meet the expectations of the target readers is more urgent. This urgent desire is expressed in the translation of the "view fusion" of the translation. Sight is the area of vision that encompasses what an understanding can see from a foothold before it begins to understand. The way a person observes and thinks about a problem is a long-established value. It is determined by factors such as ethics, aesthetic psychology, and religious beliefs. That is to say, these factors determine the foothold of the understander when he begins to understand things or phenomena, and determine the horizon of the understander. The living environment, social and cultural environment are different, and each nation has its own unique scope of vision. Translation is a special activity that expresses the meaning expressed by one language in another language. There is already some inherent information in it, and it has its own subjective tendency. Therefore, readers will actively understand and grasp the works according to their own inherent perspectives, instead of completely passive acceptance.

Therefore, when translators engage in translation activities, on the one hand, in order to understand the original text correctly and completely, we must fully consider the horizon of the source language, and examine and understand the text from the perspective of the source language. On the other hand, it is necessary to consider the comprehensibility and aesthetic requirements of the translated text from the perspective of the target language. Zhu Jianping even pointed out that translation is the fusion of horizons, and the final generation of meaning is the result of the fusion of text horizon and interpretation horizon. The "text view" here obviously refers to the view of the source text. The "interpretation horizon" is the field of view produced by the translator when interpreting the meaning of the source text in the target language. It can be seen that only the fusion of the source language text and the text field of the target language can realize the realization of the meaning of the text and ensure the smooth completion of the translation activity. Moreover, the 
higher the degree of fusion of the source text and the target text, the more successful the translation activity ${ }^{[3]}$.

\section{Translation of "Vision Fusion" trademark under the guidance of others}

\subsection{Restriction of trademark translation under the guidance of others}

The restriction of trademark translation means that in the process of trademark translation, the translator should try to make the translation the same as the original sentence, and to make the translation conform to the reading habits of the target language reader to the greatest extent, forming a kind of source language and the third language between the target languages. Trademarks are labels that are circulated around the world. In addition, trade between countries has increased in recent years, and the circulation rate of products in various countries has been continuously improved. It is possible for the producing countries and importing countries to accurately identify the relevant trademarks. The translation satisfies the needs of the target language readers on the same basis as the original sentence.

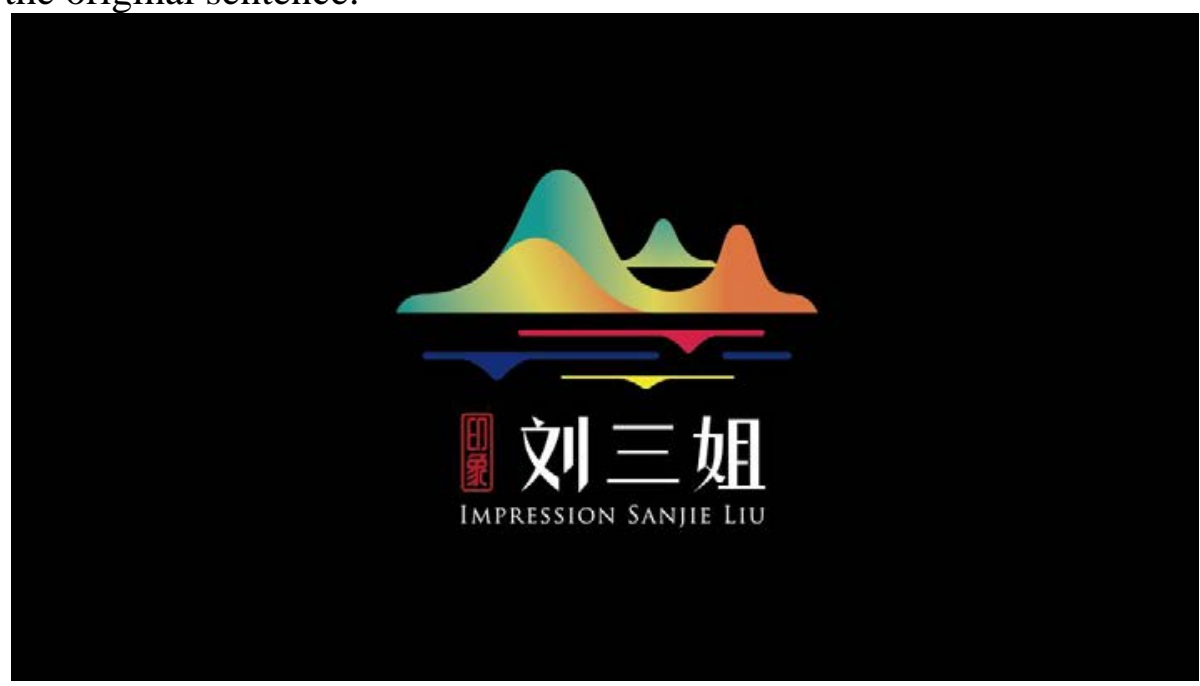

Figure 2 Impression of Liu Sanjie's Chinese and English trademarks

The trademark "Impression Liu Sanjie" of Guilin Guangwei Wenhua Tourism Culture Industry Co., Ltd. is one of the well-known trademarks announced by the Trademark Office of the State Administration for Industry and Commerce. Its English trademark is "IMPRESSION SANJIE LIU". As shown in Figure 2, it can be seen from the translation that the translation of the translation respects both the expression of Chinese and the expression of English, which satisfies the needs of English readers: the word order of the translation is the same as the original word order, which conforms to the language habits of the source language. The name of the translation also retains the combination of Chinese Pinyin, which is conducive to the understanding of Chinese readers; And its English translation also uses the authentic English format, the word "Impression" is translated directly with the English word "impression", for "Liu Sanjie" Although the translation of the title retains the Chinese Pinyin, it adopts the format of the English name, which is consistent with the language habits of the target readers. For the Chinese, Liu Sanjie is a figure in Zhuang folklore and has the reputation of " songs". Many people are more familiar with Liu Sanjie. For foreigners, Liu Sanjie is a symbol of Chinese Zhuang's song culture and represents Chinese folk. The precious intangible cultural heritage is full of strong regional characteristics. After fully considering the orientation of others, the translator chose the language hybrid translation method of English format and pinyin to translate the title of "Liu Sanjie", which not only reduced the difficulty of foreign 
tourists' understanding, but also demonstrated the internationalization of Guilin tourism, and adapted and satisfied. The emotional requirements of Chinese tourists have highlighted China's cultural characteristics, achieved good translation results, and also achieved translation goals ${ }^{[4]}$.

\subsection{The creativity of trademark translation under the guidance of others' vision}

Translation not only transforms one language into another, but also involves many aspects such as culture and customs. Therefore, the process of translation necessarily involves the fusion of culture. The language culture in the heterozygous state brings together the characteristics of the two languages and cultures. After mutual absorption and integration, the transcendence and optimization of the original culture are realized.

Under the guidance of others, in the process of translating trademarks, translators must understand the relevant cultures contained in trademarks. In translation, they can adopt the method of positive migration to integrate the target language culture into translation, so that the translation can correctly understand the trademark literals. On the basis of meaning, understand the culture of trademarks. If the literal translation according to the original trademark does not reflect the characteristics and positioning of the product well and cannot convey the culture of the superscript, the translator can choose to translate the trademark with the cultural and emotional image that is consistent with the original trademark. At this time, the translation does not implement simple superficial language translation, but a deep cultural integration. For example, the well-known trademark "Yalan" has an English translation of "AIRLAND". Whether it is literally or phonologically, the two seem to be quite different, but from a cultural perspective, the cultures of the two are similar. Wonderful. The "Ya" in Chinese trademarks is reminiscent of elegance, elegance and elegance, and "Lan" has a high quality in the hearts of Chinese people. In general, the "Yalan" trademark is left to consumers with the impression that it is "green, comfortable, high quality". The translator translates it into "AIRLAND", which refers to the military "machine drop", and is also used to refer to the integration of heaven and earth, which leads to the integration of air and the earth, meaning harmony, nature, and forever. The combination of the cultural and emotional imagery of the word in English and the commodity makes it easy for consumers to think of green harmony, harmony between man and nature, and durability. It can be seen that the Chinese trademark and the translated English trademark reflect the similar cultural sentiment and conform to the company's goal of providing consumers with comfortable, environmentally friendly and high-quality bedding. It is a good blend of the expectations of the product and the target group, and pays attention to others. Guided and realized the creativity of trademark translation ${ }^{[5]}$.

\section{Conclusions}

In general, the orientation of others in the social behavior orientation of Chinese people and the particularity of trademark translation allow translators to consciously consider the perspectives of others in trademark translation, and strive to achieve the "view fusion" of translations. "Vision Fusion" regulates the whole process of trademark translation activities. On the one hand, it has a restrictive effect on trademark translation, which must conform to the "vision" of the target population and be recognized by them. On the other hand, it plays a creative role, inspiring translators to create more effective trademark translations, "discard" original trademarks, and create novel, international and meaningful "new" trademarks.

\section{References}

[1] Wang Xueshuang. Looking at English-Chinese Trademark Translation from the Perspective of Skopos Theory[J]. 
Journal of Tianjin Agricultural University, 2010, 17(3): 52-54.

[2] Chang Jinjin. The style characteristics of English translation legislative texts from the perspective of functional stylistics [D]. Guangdong University of Foreign Studies, 2013.

[3] Liu Yurong. Equivalent Translation Theory and Translation of English and Chinese Trademark Words[J]. Journal of Jiangxi Normal University (Philosophy and Social Sciences), 2004, 37(2): 85-88.

[4] Zhang Zhili. Composition and Translation Principles of Chinese and English Trademark Names[J]. Journal of Shenyang Agricultural University, Social Science Edition, 2008, 10(1): 98-100.

[5] Song Pengfei. On the Translation of Trademarks from the Perspective of Functionalist Translation Theory[J]. Journal of Beijing Electric Power College: Social Sciences Edition, 2012, 29(7). 